\title{
NODULE DETECTION IN DIGITAL CHEST RADIOGRAPHY: PART OF IMAGE BACKGROUND ACTING AS PURE NOISE
}

\author{
Magnus Båth $^{1 *}$, Markus Håkansson ${ }^{1}$, Sara Börjesson ${ }^{1}$, Susanne Kheddache $^{2}$, Anna Grahn ${ }^{3}$, \\ François O. Bochud ${ }^{4}$, Francis R. Verdun ${ }^{4}$ and Lars Gunnar Månsson ${ }^{1}$ \\ ${ }^{1}$ Department of Medical Physics and Biomedical Engineering, Sahlgrenska University Hospital, \\ SE-413 45 Göteborg, Sweden \\ ${ }^{2}$ Department of Radiology, Sahlgrenska University Hospital, SE-413 45 Göteborg, Sweden \\ ${ }^{3}$ Department of Radiation Physics, Malmö University Hospital, SE-205 02 Malmö, Sweden \\ ${ }^{4}$ University Institute for Applied Radiophysics, CH-1007 Lausanne, Switzerland
}

\begin{abstract}
There are several factors that influence the radiologist's ability to detect a specific structure/lesion in a radiograph. Three factors that are commonly known to be of major importance are the signal itself, the system noise and the projected anatomy. The aim of this study was to determine to what extent the image background acts as pure noise for the detection of subtle lung nodules in five different regions of the chest. A receiver operating characteristic (ROC) study with five observers was conducted on two different sets of images, clinical chest X-ray images and images with a similar power spectrum as the clinical images but with a random phase spectrum, resulting in an image background containing pure noise. Simulated designer nodules with a full-width-at-fifth-maximum of $10 \mathrm{~mm}$ but with varying contrasts were added to the images. As a measure of the part of the image background that acts as pure noise, the ratio between the contrast needed to obtain an area under the ROC curve of 0.80 in the clinical images to that in the random-phase images was used. The ratio ranged from 0.40 (in the lateral pulmonary regions) to $\mathbf{0 . 8 3}$ (in the hilar regions) indicating that there was a large difference between different regions regarding to what extent the image background acted as pure noise; and that in the hilar regions the image background almost completely acted as pure noise for the detection of $10 \mathrm{~mm}$ nodules.
\end{abstract}

\section{INTRODUCTION}

In the recent years, a lot of effort has been made to investigate the components that disturb the detection of pathology for different X-ray examinations, such as mammography ${ }^{(1-3)}$, chest radiography ${ }^{(4,5)}$ and lumbar spine radiography ${ }^{(6)}$. In several of these studies, it has been shown that in many cases system noise (including quantum noise) is not the major component disturbing the detection of details but the projected anatomy itself. Bochud et al. ${ }^{(1)}$ investigated this effect in mammography and found that it was strongly dependent on the combination of the type of image background and the type of signal. For one combination, the performance of the human observers was equal to that of model observers in images containing only system noise (of the same amount as in the real images), whereas for another combination, the human observers did not perform better in the real images than model observers did in images that had the same power spectrum as the real images but random phase spectrum, thereby containing only pure noise ${ }^{(7)}$. The conclusion that can be drawn from these results is that for the first combination the projected anatomy did not disturb the detection at all, whereas for the other the projected anatomy disturbed the observer as if it had been pure noise.

*Corresponding author: magnus.bath@vgregion.se
The aim of the present study was to determine to what extent the image background acts as pure noise for the detection of lung nodules in chest radiography. Such an investigation is of importance for several reasons: it is of interest for the optimisation of beam quality and dose level, it gives information about to what extent the human observer can 'prewhite' the image - indicating that the observer is capable of distinguishing a fixed pattern associated with the image background and remove the pattern so that the detection task is not disturbed by the pattern ${ }^{(8)}$ and it explores the limits of projection radiography.

The study described in the present paper is one of four studies constituting a larger investigation (the RADIUS chest trial) thoroughly examining various aspects of nodule detection in digital chest radiography. The RADIUS chest trial, conducted by members of the Radiological Imaging Unification Strategies (RADIUS) group (a European-wide research group financed by the European Commission), is introduced by Båth et al. ${ }^{(9)}$, and a review of recent research leading to the investigation is provided. The investigation is summarised in a paper by Håkansson et al. ${ }^{(10)}$, in which the results from the four studies are analysed in a joint manner. Details of the studies constituting the investigation are described in four papers, of which the present paper, examining to what extent the image background acts as pure noise, is one. The other three papers deal with the effects of nodule location ${ }^{(11)}$, system noise $^{(12)}$ and anatomical noise ${ }^{(13)}$. 


\section{MATERIALS AND METHODS}

\section{Introductory remarks}

In the paper by Håkansson et al. ${ }^{(11)}$, where the effect of nodule location on detection was studied, the chest radiograph was divided into six different regions, each region being somewhat uniform in terms of difficulty. Five of the six regions were finally examined in that study, and as a measure of detectability, the detail contrast needed for obtaining an area under the receiver operating characteristic (ROC) curve $\left(A_{\mathrm{z}}\right)$ of $0.80\left(\mathrm{C}_{0.8}\right)$ for simulated nodules of diameter $10 \mathrm{~mm}$ was used. The same approach was used in the present work. As a measure of the part of the image background that acts as pure noise, the ratio between $\mathrm{C}_{0.8}$ for clinical images and $\mathrm{C}_{0.8}$ for images with similar power spectrum but random phase (thereby containing only noise) was used. The five regions analysed by Håkansson et al. ${ }^{(1)}$ (see Figure 1 and Table 1) were investigated separately.

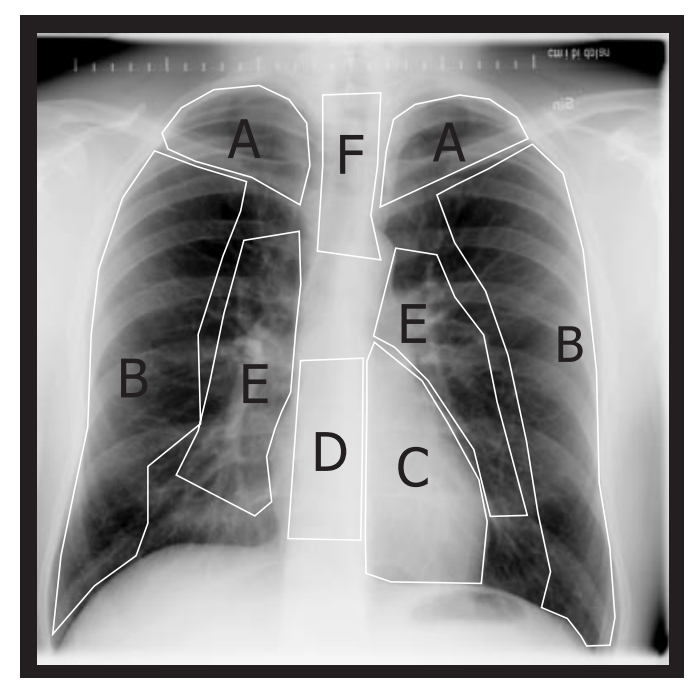

Figure 1. Graphical representation of the regions used in the study. Note: Region A was excluded from the study.

Table 1. Description of the five regions examined in the study.

\begin{tabular}{ll}
\hline Region & Description \\
\hline B & The lateral pulmonary regions \\
C & The retrocardial region \\
D & The lower mediastinal region (the spine) \\
E & The hilar regions \\
F & The upper mediastinal region (the trachea) \\
\hline
\end{tabular}

\section{Production of random-phase images}

To determine the power spectrum of each of the five regions, regions of interest (ROIs) of size $128 \times 128$ pixels were extracted from 30 clinical chest radiographs collected using the computed radiography system Fuji FCR 9501 (Fuji Photo Film, Tokyo, Japan). (The same 30 images were used to determine $\mathrm{C}_{0.8}$ for the clinical image backgrounds by Håkansson et al. ${ }^{(1)}$.) The size of the ROI was a compromise between the negative effects of using small ROIs (edge artefacts and spectral leakage) and a goal to include as much as possible of the anatomy (the sizes of the regions were such that large ROIs would not fit in the regions). The ROIs were positioned next to each other resulting in between 100 and 300 realisations of the anatomy for each region.

Calculating the power spectrum of a noise process with a strong correlation between pixels leads to large edge artefacts. A common method of solving this problem is to use windowing ${ }^{(14,15)}$. However, since the ROIs were as small as they were, the effects of the window itself became dominant (the values of the low-frequency components were strongly affected by the windowing). Instead, a different method was used. The edge artefact is caused by the discontinuity introduced by the use of the discrete Fourier transform (DFT). Simply speaking, the DFT assumes a periodicity that is not present in the anatomical cut-outs (the cut-out cannot be positioned to produce wallpaper). However, by mirroring each ROI to produce a larger $256 \times 256 \mathrm{ROI}$, a periodicity was introduced. An edge artefact was still present, since the mirroring itself introduced a small discontinuity. However, the magnitude of this artefact was much lower than that produced when no mirroring was being used.

Owing to the differences in exposure, patient thickness and anatomy itself, the difference in average exposure between ROIs belonging to a specific region was large. To increase the precision of the power spectrum determination, the normalised power spectra of the ROIs belonging to each region were therefore averaged. (The normalisation was achieved by dividing the power spectrum with the square of the mean pixel value in each ROI. Hence, since the projection of anatomy leads to a deterministic signal, the normalised power spectrum of the reproduced anatomy is independent of the dose level.)

It was decided that although the mirroring reduced the artefact from the finite ROI size, the artefact was still unacceptably large. The twodimensional power spectra were, therefore, radially averaged. The artefact was mainly influencing data along the axes of the two-dimensional power spectra and hence they were excluded.

It has been shown that the power spectrum of several types of background can be described using 


\section{BATH ET AL.}

Table 2. The parameters used to fit the sum of two power-law functions to the measured power spectra for the five regions.

\begin{tabular}{lcccc}
\hline Region & $\alpha_{1}$ & $\beta_{1}$ & $\alpha_{2}$ & $\beta_{2}$ \\
\hline B & $1.8 \times 10^{-6}$ & 3.2 & $1.9 \times 10^{-5}$ & 0.5 \\
$\mathrm{C}$ & $6.0 \times 10^{-6}$ & 3.1 & $4.5 \times 10^{-5}$ & 0.4 \\
$\mathrm{D}$ & $3.0 \times 10^{-6}$ & 3.2 & $7.0 \times 10^{-5}$ & 0.5 \\
$\mathrm{E}$ & $2.0 \times 10^{-5}$ & 3.4 & $1.4 \times 10^{-5}$ & 0.5 \\
$\mathrm{~F}$ & $1.0 \times 10^{-5}$ & 3.3 & $4.0 \times 10^{-5}$ & 0.7 \\
\hline
\end{tabular}

a single power-law function ${ }^{(2,14,16)}$, at least for low frequencies. In the present work, a sum of two power-law functions was needed; probably due to a relative increase in the system noise components at high frequencies. The determined one-dimensional power spectra were, therefore, fitted to an analytical function of the form:

$$
N(u)=\frac{\alpha_{1}}{u^{\beta_{1}}}+\frac{\alpha_{2}}{u^{\beta_{2}}}
$$

The values of the components in Equation 1 for the different regions can be found in Table 2. The random-phase images were produced by creating complex arrays of the desired size $(512 \times 512$ pixels were used) - corresponding to the random-phase images in the frequency domain - and setting the magnitude of each frequency value according to the analytical expression. Random phase with a uniform probability density between 0 and $2 \pi$ was addressed to the frequency values. (For the zero frequency, the phase was always set to 0 , and for the Nyquist frequencies along the axes and along the diagonal of the phase was randomly set to 0 or $\pi$.) To obtain real images, the symmetry of the phase spectrum, $\Phi$, was odd $[\Phi(u)=-\Phi(-u)]$. By Fourier transformation, real images were obtained. To validate the analytical fit, one-dimensional power spectra were calculated for the random-phase images in the same way as was done for the clinical images (Figure 2). In Figures 3 and 4 , examples of cut-outs of clinical images and the corresponding random-phase images are presented for two of the five regions used.

\section{Study set-up}

For each region, 180 unique $512 \times 512$ randomphase images were produced. With five different regions, this led to a total of 900 images. In half of these, a simulated 'designer' nodule ${ }^{(17)}$ was inserted. The full-width-at-fifth-maximum was $10 \mathrm{~mm}$. Three different contrast levels were used, each level being $22.5 \%$ higher than the previous. The baseline contrast was chosen individually for each region, based on the results of pilot studies.

An ROC study was conducted with five observers (two radiologists and three medical physicists).
The study was conducted in a completely digital environment with both image presentation and scoring conducted using software developed in-house ${ }^{(18)}$. Each observer viewed all images in a unique random order. A five-graded scale was used for the confidence of each positive/negative decision. The data from all five observers were pooled, and the software ROCFIT (C. E. Metz, University of Chicago) was used to calculate the $A_{\mathrm{z}}$ for each combination of region and detail contrast. As a measure of detectability, $\mathrm{C}_{0.8}$ was used. The measure was obtained from a logarithmic fit of $A_{\mathrm{z}}$ vs. contrast (see Figure 5). Values of $\mathrm{C}_{0.8}$ for the clinical images were obtained from the ROC study conducted in a similar way described by Håkansson et al. ${ }^{(11)}$. The same observers were used in both studies.

An estimation of the uncertainty in $\mathrm{C}_{0.8}$ for a specific region was obtained in the following way. For each of the three contrast levels, the standard deviation of the $A_{\mathrm{z}}$ was obtained from ROCFIT. The square root of the sum of squares of the standard deviations was then divided by 3 (the number of independent observations for each logarithmic fit). The number obtained in this way, reflecting the uncertainty in $A_{\mathrm{z}}$ for a specific contrast, was then transformed to the uncertainty in contrast $\left(\mathrm{C}_{0.8}\right)$ for a specific $A_{\mathrm{z}}(0.80)$ through the logarithmic fit.

\section{RESULTS}

The estimated relative uncertainty in the $\mathrm{C}_{0.8}$ was $\sim 3 \%$ both for the random-phase images and for the clinical images, leading to an average relative uncertainty of $4 \%$ for the obtained ratio between the $\mathrm{C}_{0.8}$ values for the clinical images and the random-phase images.

The detail contrast needed for obtaining an $A_{\mathrm{z}}$ of 0.80 in the different regions for the random-phase images is presented in Table 3 . The ratio between the contrast needed in the clinical images and the random-phase images ranged between 0.40 (Region B) to 0.83 (Region E), indicating that Region B was the region for which the image background acted least as pure noise whereas vice versa for Region E. Region D was both the easiest region (smallest contrast needed in the clinical images) and the region with the smallest amount of variations (smallest contrast needed in the random-phase images) whereas the opposite was true for Region E.

\section{DISCUSSION}

Many interesting results can be found in Table 3 if the data are analysed in a closer manner. The $\mathrm{C}_{0.8}$ values for the clinical images describe the difficulty of the regions, whereas the $\mathrm{C}_{0.8}$ values for the random-phase images describe the amount of variations in the regions - regardless to what extent the 

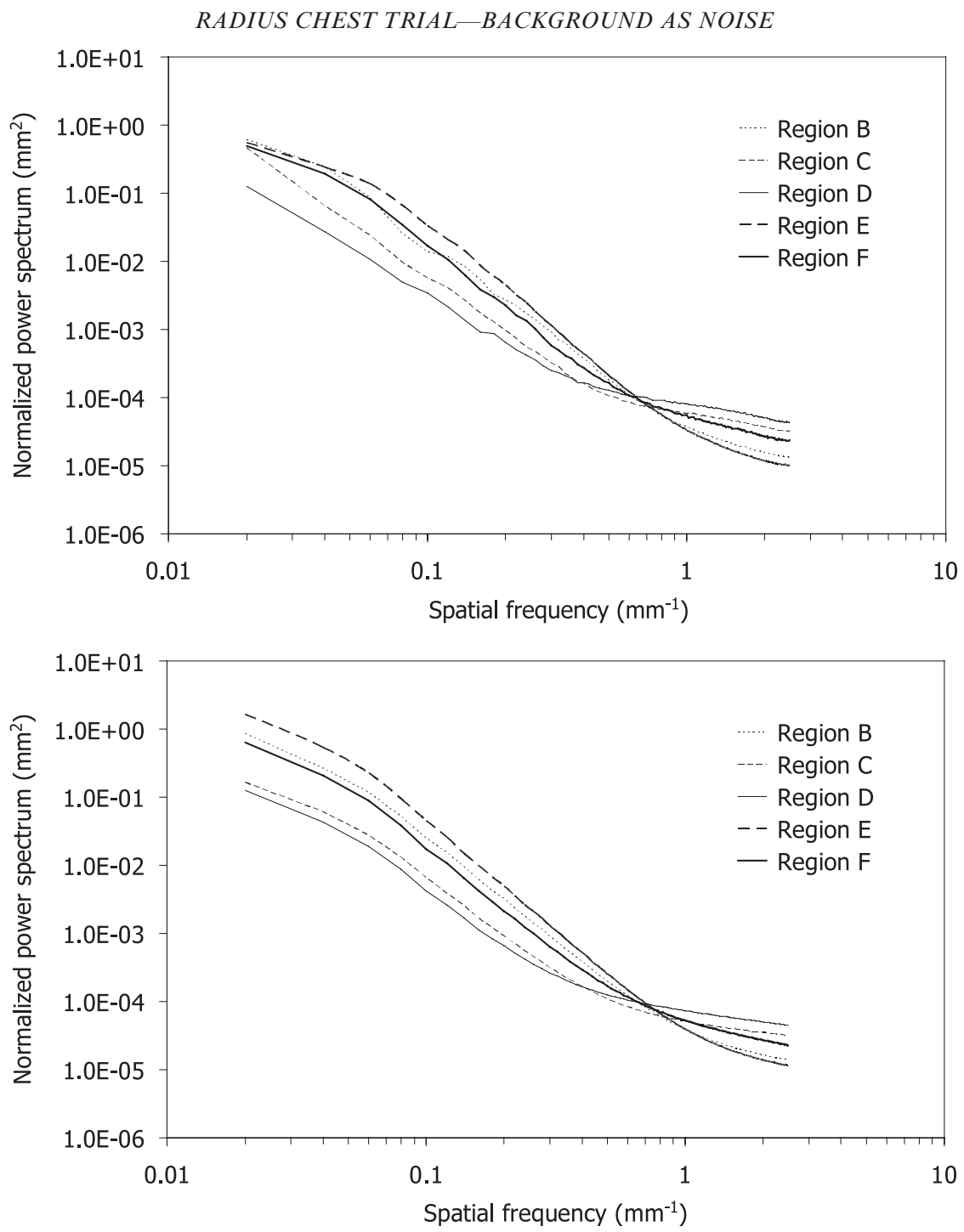

Figure 2. Measured one-dimensional power spectra of the clinical images (upper panel) and the generated random-phase images (lower panel).

variations in the clinical images disturb the observer (acts as pure noise). The ratio between the $\mathrm{C}_{0.8}$ values for the clinical images and the random-phase images is a measure of to what extent the image background in the regions acts as pure noise. Region $\mathrm{E}$ (hilar regions) has the highest $\mathrm{C}_{0.8}$ values for both types of images, indicating that it is not only the most difficult region, but also the region which to a largest extent acts as pure noise for the detection of the inserted nodules, which can easily be understood. There are large variations in the region (leading to a large contrast needed for detection when the information is transformed into noise) and the structures in the region can easily be mistaken for nodules, e.g. overlapping of large vessels (leading also to a large contrast needed for detection in the clinical images). Region B is completely different. The contrast needed in the random-phase images is large but only a small contrast is needed for the clinical images. This is due to the large impact of the ribs (leading to a lot of noise power in the randomphase images but not strongly affecting the detection in the clinical images). Furthermore, Region B to a smaller extent than Region E contains structures that can be mistaken for the $10 \mathrm{~mm}$ nodules used in the study. Region D was by far the easiest region, both for the clinical images and for the randomphase images. 

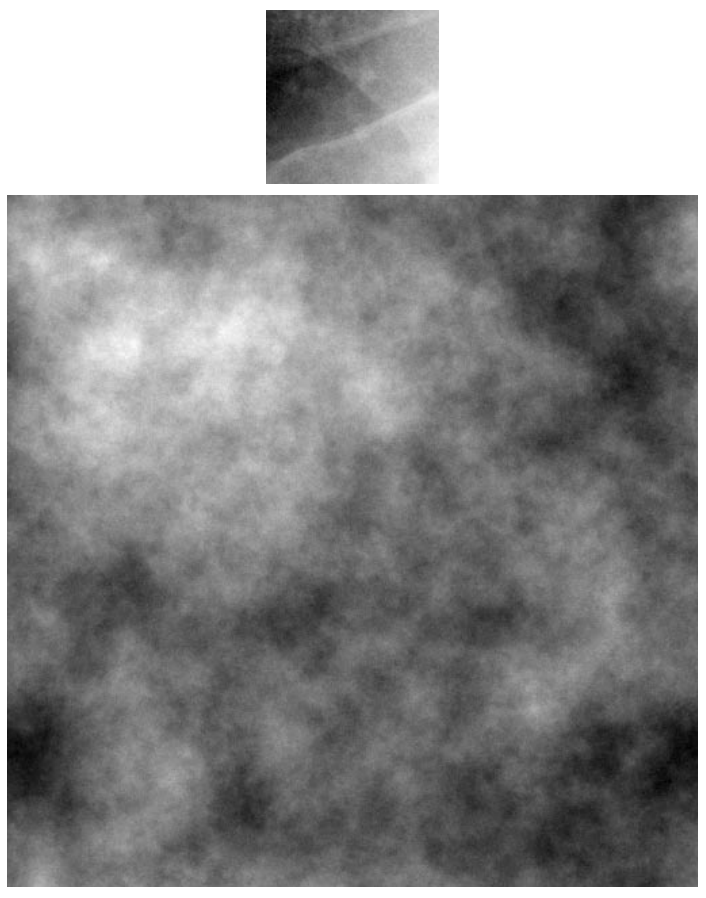

Figure 3. Example of a $128 \times 128$ pixels cut-out (upper panel) used for producing $512 \times 512$ pixels random-phase images (lower panel) for Region B.

It should be noted that the ratio between the two $\mathrm{C}_{0.8}$ values does not have a maximum of unity. An image could be produced that only contains overlapping of nodules of the same shape and size as the one searched for (the excess frequency power being used to produce a certain image background), in which case the task would become extremely difficult and result in a higher value of $\mathrm{C}_{0.8}$ than the randomphase image containing pure noise. The randomphase images are merely used to normalise the contrast needed for the detection to that of images in which the frequency power is randomly positioned in the images. The term 'acts as pure noise' describes both to what extent the image background resembles the signal and to what extent the observer is confused by the background. An experienced observer may know that the overlapping of certain structures results in a nodule-like appearance and disregard from it, whereas an inexperienced observer erroneously detects it as a signal. All the observers in the present study were experienced in evaluating chest images (no significant difference in performance in the clinical images was found between the radiologists and the medical physicists $\left.{ }^{(11)}\right)$, nevertheless the contrast ratios were surprisingly high.

Owing to the system noise (including quantum noise and detector noise), also the clinical images contain pure noise, indicating that even in the ideal

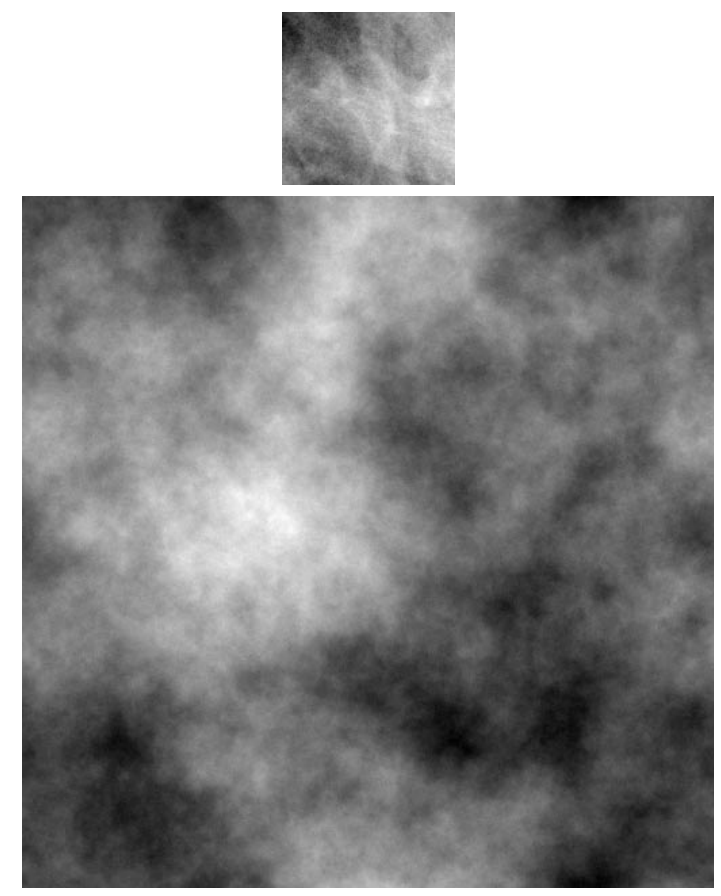

Figure 4. Example of a $128 \times 128$ pixels cut-out (upper panel) used for producing $512 \times 512$ pixels random-phase images (lower panel) for Region E.

case of an anatomical background completely recognised by the observer and not resembling the signal at all, a contrast ratio larger than zero would be obtained. Since the system noise varies between the regions due to, for example, differences in attenuation, the magnitude of this effect is slightly different for different regions. Refer to Håkansson et al. ${ }^{(12)}$ for details on the effect of the system noise in the different regions and to the papers giving an overview of the RADIUS chest trial ${ }^{(9,10)}$ for a complete analysis.

It should be noted that the detail contrast levels presented in Table 3 refer to image contrast (although not to display image contrast which could be set arbitrarily) and not to object contrast. Owing to differences in the amount of scattered radiation in the different regions, the object contrast needed for detection is different. This difference is further discussed by Håkansson et al. ${ }^{(11)}$, and the effect of nodule location on detection is thoroughly examined.

For each region, the power spectra of the clinical images and of the random-phase images were not identical, but based on the fact that the difference between the power spectra for the different regions was larger than the difference between the randomphase power spectrum and the anatomical power spectrum for a specific region, it was decided that 
RADIUS CHEST TRIAL-BACKGROUND AS NOISE

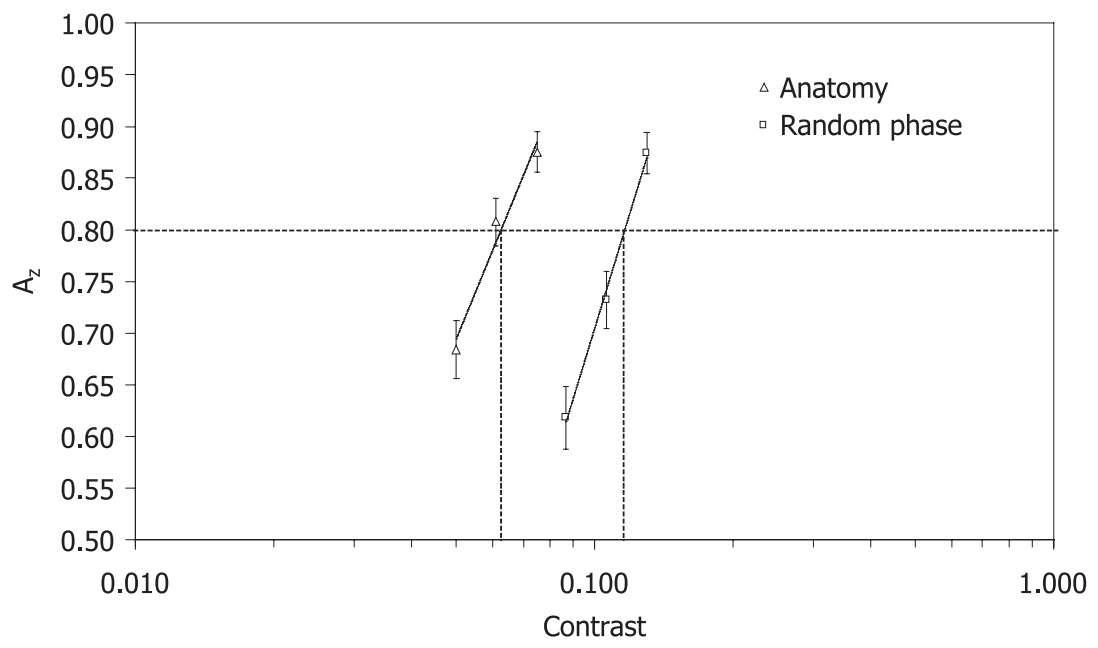

Figure 5. $A_{\mathrm{z}}$ vs. detail contrast for the clinical images and the random-phase images for Region D. Error bars are $\pm 1 \mathrm{SD}$. $\mathrm{C}_{0.8}$ is obtained from a logarithmic fit.

Table 3. $\mathrm{C}_{0.8}$ for the clinical images and for the random-phase images for the five regions as well as the ratio between the two values.

\begin{tabular}{lccc}
\hline Region & Anatomy & Random phase & Ratio \\
\hline B & 0.101 & 0.257 & 0.40 \\
C & 0.101 & 0.136 & 0.74 \\
D & 0.063 & 0.116 & 0.54 \\
E & 0.258 & 0.310 & 0.83 \\
F & 0.141 & 0.225 & 0.63 \\
\hline
\end{tabular}

Estimated relative uncertainty: anatomy, 3\%; random phase, $3 \%$; and ratio, $4 \%$

the accuracy was acceptable. Nevertheless, comparing the two kinds of spectra, it is possible that the $\mathrm{C}_{0.8}$ for random-phase images with the same power spectrum as the anatomical Region $\mathrm{C}$ is somewhat underestimated with the random-phase images used, whereas the opposite might be true for Region E. However, the power spectra differences are only substantial at the absolutely lowest frequencies, which probably do not influence the detection of the nodules to a large extent. Furthermore, the anatomical power spectrum of Region $\mathrm{E}$ is definitely higher than that of Region B, for which the difference between the anatomical power spectrum and the randomphase power spectrum is small, and the determined $\mathrm{C}_{0.8}$ for the random-phase images is only $20 \%$ higher for Region $\mathrm{E}$ than for Region $\mathrm{B}$, indicating that the possible overestimation is $<20 \%$.

To fully explain the results presented in the present paper, the effects of nodule location ${ }^{(11)}$, system noise ${ }^{(12)}$ and anatomical noise ${ }^{(13)}$ must also be taken into account. As mentioned previously, two summarising papers ${ }^{(9,10)}$ aim at thoroughly investigating these effects in combination with the results presented in this paper, and the reader is referred to those papers for an overall picture of nodule detection in digital chest radiography.

\section{CONCLUSIONS}

There is a large difference between different regions in the chest radiograph regarding to what extent the image background acts as pure noise. In the hilar regions, the image background almost completely acts as pure noise for the detection of $10 \mathrm{~mm}$ nodules.

\section{ACKNOWLEDGEMENTS}

The authors would like to thank Oleg Tischenko for stimulating discussions, and Ase Allansdotter Johnsson, Agneta Flinck and Jenny Vikgren for reading the images. This work was supported by a grant from the European Commission (contract no. FIGM-CT-2000-00036).

\section{REFERENCES}

1. Bochud, F. O., Valley, J.-F., Verdun, F. R., Hessler, C. and Schnyder, P. Estimation of the noisy component of anatomical backgrounds. Med. Phys. 26, 1365-1370 (1999).

2. Burgess, A. E., Jacobson, F. L. and Judy, P. F. Human observer detection experiments with mammograms and power-law noise. Med. Phys. 28, 419-437 (2001).

3. Huda, W., Ogden, K. M., Scalzetti, E. M., Dudley, E. F. and Dance, D. R. How do radiographic techniques affect mass lesion detection performance in digital mammography. Proc. SPIE 5372, 372-382 (2004).

4. Samei, E., Flynn, M. J. and Eyler, W. R. Detection of subtle lung nodules: relative influence of quantum and 


\section{BAंTH ET AL.}

anatomic noise on chest radiographs. Radiology $\mathbf{2 1 3}$, 727-734 (1999).

5. Keelan, B. W., Topfer, K., Yorkston, J., Sehnert, W. J. and Ellinwood, J. S. Relative impact of detector noise and anatomical structure on lung nodule detection. Proc. SPIE 5372, 230-241 (2004).

6. Tingberg, A., Båth, M., Håkansson, M., Medin, J., Sandborg, M., Alm-Carlsson, G., Mattsson, S. and Månsson, L. G. Comparison of two methods for evaluation of image quality of lumbar spine radiographs. Proc. SPIE 5372, 251-262 (2004).

7. Burgess, A., Jacobson, F. and Judy, P. Mass discrimination in mammography: experiments using hybrid images. Acad. Radiol. 10, 1247-1256 (2003).

8. Samei E., Eyler, W. and Baron, L. Effects of anatomical structure on signal detection. In Handbook of Medical Imaging. Vol. 1. Physics and Psychophysics. Beutel, J., Kundel, H.L. and Van Metter, R.L., Eds. (Bellingham: SPIE Press) pp. 655-682 (2000).

9. Båth, M., Håkansson, M., Börjesson, S., Kheddache, S., Grahn, A., Ruschin, M., Tingberg, A., Mattsson, S. and Månsson, L. G. Nodule detection in digital chest radiography: introduction to the RADIUS chest trial. Radiat. Prot. Dosim. 114(1-3), 85-91 (2005).

10. Håkansson, M., Båth, M., Börjesson, S., Kheddache, S., Grahn, A., Ruschin, M., Tingberg, A., Mattsson, S. and Månsson, L. G. Nodule detection in digital chest radiography: summary of the RADIUS chest trial. Radiat. Prot. Dosim. 114(1-3), 114-120 (2005).

11. Håkansson, M., Båth, M., Börjesson, S., Kheddache, S., Flinck, A., Ullman, G. and Månsson, L. G. Nodule detection in digital chest radiography: effect of nodule location. Radiat. Prot. Dosim. 114(1-3), 92-96 (2005).

12. Håkansson, M., Båth, M., Börjesson, S., Kheddache, S., Allansdotter Johnsson, А. and Månsson, L. G. Nodule detection in digital chest radiography: effect of system noise. Radiat. Prot. Dosim. 114(1-3), 97-101 (2005).

13. Båth, M., Håkansson, M., Börjesson, S., Hoeschen, C., Tischenko, O., Kheddache, S., Vikgren, J. and Månsson, L. G. Nodule detection in digital chest radiography: effect of anatomical noise. Radiat. Prot. Dosim. 114(1-3), 109-113 (2005).

14. Bochud, F. O., Abbey, C. K. and Eckstein, M. P. Visual signal detection in structured background. III. Calculation of figures of merit for model observers in statistically nonstationary backgrounds. J. Opt. Soc. Am. 17, 193-205 (2000).

15. Aufrichtig, R., Su, Y., Cheng, Y. and Granfors, P. R. Measurement of the noise power spectrum in digital $x$-ray detectors. Proc. SPIE 4320, 362-372 (2001).

16. Bochud, F. O., Abbey, C. K. and Eckstein, M. P. Further investigation of the effect of phase spectrum on visual detection in structured backgrounds. Proc. SPIE 3663, 273-281 (1999).

17. Burgess, A. E., Li, X. and Abbey, C. K. Nodule detection in two component noise: toward patient structure. Proc. SPIE 3036, 2-13 (1997).

18. Börjesson, S. and 10 others. A software tool for increased efficiency in observer performance studies in radiology. Radiat. Prot. Dosim. 114(1-3), 45-52 (2005). 